\title{
Two-State Passive Mode-Locking of Quantum Dot Semiconductor Lasers: Classical State Scenario and Novel Reverse State Dynamics
}

\author{
Stefan Breuer, Member, IEEE, Mattia Rossetti*, Wolfgang Elsässer, Senior Member, IEEE, Lukas \\ Drzewietzki, Paolo Bardella*, Ivo Montrosset*, Member, IEEE, Michel Krakowski**, and Mark \\ Hopkinson, Member, IEEE*** \\ Technische Universität Darmstadt, Schlossgartenstraße 7, 64289 Darmstadt, Germany \\ *Politecnico di Torino, Corso Duca degli Abruzzi 24, 10129 Torino, Italy \\ **Alcatel Thales, III-V Lab, Route départementale 128, 91767 Palaiseau, France \\ ***University of Sheffield, Mappin Street, Sheffield, S1 3JD, United Kingdom \\ Tel: (++49) 6151 16-4086, Fax: (++49)6151 16-3022, e-mail: stefan.breuer@physik.tu-darmstadt.de
}

\begin{abstract}
We present both experimental and theoretical investigations of the so-called reverse emission state dynamics in a two-section InAs/InGaAs Quantum Dot (QD) laser. In contrast to the classical state scenario, we demonstrate by properly designing the laser cavity and the QD active region, a reversal of the emission state transition: At a certain gain current Excited-state (ES) lasing and mode-locking (ML) starts first and then, with increasing gain current, a transition to simultaneous ES and ground-state (GS) ML takes place. This enables a novel approach to wavelength-switching of the mode-locked pulses over a range of $63 \mathrm{~nm}$ : the realization of a two-section QD laser with a resistor Self-Electro-optic Effect Device (SEED) configuration. These results are reviewed together with the state-of-the-art realization of InAs/InGaAs two-section QD lasers operating in two-state ML regime .

Keywords: two-state and dual-wavelength emission, passive mode-locking, optical wavelength switching, quantum dot laser emission hierarchy, two-section quantum dot semiconductor laser, stable picosecond pulse generation, quantum dot resistor Self-Electro-optic Effect Device (SEED).
\end{abstract}

\section{INTRODUCTION}

Passively mode-locked semiconductor lasers based on QDs exhibit a particular interesting dynamical behavior due to the existence of two quantized states, GS and ES, in the dot energy scheme [1-3]. Simultaneous two-state lasing and mode-locking in InAs/InGaAs two-section QD lasers, i.e. the simultaneous lasing and the generation of stable ML pulses from both GS and ES QD transitions, has already been demonstrated and experimentally investigated in detail; in those devices, with increasing gain current, a transition from GS to ES emission and a coexistence regime at intermediate currents with lasing was found and possible ML from both transitions was achieved [4-6].

In this contribution, the very recently reported reverse emission state transition in two-section QD lasers is instead discussed, i.e. the transition from stable ES ML to stable ML on both GS and ES by increasing the gain current $[7,8]$. This particularly fascinating emission dynamics is obtained in a properly designed QD structure, allowing to achieve a stable dual-wavelength ML regime over a wide range of applied currents. Simultaneity of the stable ES and GS ML is experimentally demonstrated and simulations substantially confirm these experimentally observed results.

SEED based on quantum well structures has been proposed and studied in depth in the 80s and 90s with respect to their applications in switching, bi-stability and modulation $[9,10]$. Here, this concept is applied to a two-section QD laser structure. By controlling losses for both QD states, a complete emission-state control, i.e. wavelength-switching between $1207 \mathrm{~nm}$ and $1270 \mathrm{~nm}$, over a range of $63 \mathrm{~nm}$, by simply tuning the resistance value of a variable resistor across the SA section, is demonstrated.

\section{NOVEL DEVICE DESIGN AND EXPERIMENTAL SET-UP}

A possible route to obtain two-state emission in a two-section QD laser is to achieve a condition in which the GS net modal gain saturates at a value slightly smaller than the sum of saturable and non-saturable cavity losses $[8,11]$. Such a condition can be satisfied by properly designing the QD laser cavity, either decreasing the cavity length or, additionally, reducing the laser facet reflectivities [4-6]. Complementary to these approaches, the specific properties of the QD active medium can also be designed to reduce the maximum net modal gain achievable at the GS wavelengths: here, two-state emission is in fact realized by reducing the number of QD layers and, in particular, by strongly chirping their characteristic emission wavelengths $[7,8]$. To implement this novel design approach, a dot-in-a-well (DWELL) structure with six DWELL layers consisting of two repeats of three differently chirped layers was realized. In these layers, InAs dots, grown by Stranski-Krastanov process via MBE, are embedded in an InGaAs Quantum Well (QW) which forms the DWELL structure. By modifying the Indium content of the three QWs, a broad gain bandwidth in the region of $>80 \mathrm{~nm}$, compared to the (50-60) nm 
bandwidth of a single DWELL layer, is achieved [12]. The gain section is processed into a ridge waveguide of $3 \mathrm{~mm}$ length and $3 \mu \mathrm{m}$ width, the saturable absorber (SA) section is $0.3 \mathrm{~mm}$ long. The facets are left as cleaved.

To experimentally investigate the reverse emission state transition ML dynamics, a DC current source is used to drive the gain section and a DC voltage source provides the reverse bias voltage to the SA section. In the investigated structure only a zero Volt bias is applied to the SA section. To exploit the resistor SEED configuration on a two-section QD laser, the SA section is instead electrically shortened via an external variable resistor $(2 \mathrm{k} \Omega)$. State-resolved measurements are performed applying a set of interference filters. An optical power meter monitors the average laser output power, an optical spectrum analyzer is used to record optical spectra. A second harmonic generation (SHG) intensity auto-correlation technique is applied to measure spectrally resolved optical pulse widths by setting the correct crystal angle. A high-speed InGaAs PIN detector and a broad-band electrical spectrum analyzer were used to perform RF analysis, to check for the onset of ML and determine the effective pulse repetition frequencies for GS and ES as well as to verify the temporal simultaneity of the two-state ML. The laser structure is mounted p-side up on a temperature-controlled laser submount, stabilized to $20^{\circ} \mathrm{C}$.

\section{TIME-DOMAIN TRAVELLING-WAVE RATE-EQUATION MODEL}

To model and finally fully understand the complex carrier dynamics involved in the novel reverse emission state transition scenario, a finite-difference time-domain travelling-wave model is developed and applied $[11,13,14]$. Appropriate propagation equations for the forward and backward travelling power that describe the evolution of the optical power along the laser cavity at both GS and ES characteristic transition wavelengths, are solved in the time domain via a finite-difference approach. According to [15], additional effects such as finite gain spectral bandwidth, self-phase modulation and dispersion are neglected in the model, assuming that the gain/absorption dynamics in the gain and SA section are the main factor leading to the realized ML regime. The evolution of the GS and ES gain and absorption dynamics in each longitudinal section of the cavity is obtained by solving a dedicated set of rate-equations in the excitonic approach. Appropriate equations for carriers in the separate confinement heterostructure, in the wetting layer and in the QD confined states are considered. Properly modified and voltage dependent rate equations modeling the carrier dynamics in the reversely-biased SA section are introduced in the model, as described in detail in [8].

\section{EXPERIMENTAL AND SIMULATION RESULTS}

Reversing the emission state transition by grounding the SA is most clearly visualized in the evolution of the experimentally obtained optical spectra. Fig. 1 exemplarily depicts four spectra obtained at 105, 120, 130 and $142 \mathrm{~mA}$, respectively, and at a constant applied reverse bias of zero volt. A spectral separation of the quantized states of $63 \mathrm{~nm}$ is indicated. As shown in Fig. 1, emission starts at $108 \mathrm{~mA}$ first on the energetically higher ES

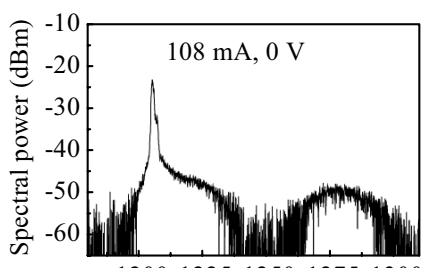

12001225125012751300

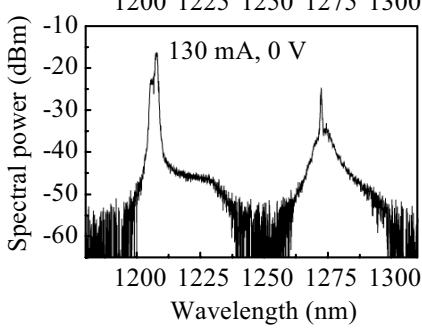

Fig. 1. Two-section QD laser reverse emission state dynamics: Optical spectra evolution with increasing gain current and at an absorber voltage of zero Volt (experimental results).
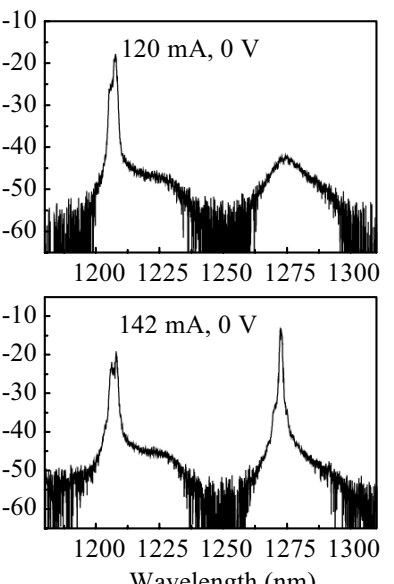

$$
\text { Fig. }
$$

RF beat spectra for ES and dual-state ML. The inset shows the low-frequency beat domain.

the gain current, at $120 \mathrm{~mA}$, GS amplified spontaneous emission increases until, at $130 \mathrm{~mA}$, emission on the GS at $1270 \mathrm{~nm}$ sets in while ES lasing persists. At $142 \mathrm{~mA}$, both states coexist, exhibiting nearly equal spectral power in both states. RF beats, the effective repetition rate of the picosecond pulses, obtained at $128 \mathrm{~mA}$ and $132 \mathrm{~mA}$ are depicted in Fig. 2. A clear signature of the temporal simultaneity of GS and ES ML is confirmed by the occurrence of a four-wave mixing (FWM) signal between GS and ES as expressed by frequency sidebands at $11.89 \mathrm{GHz}$ and $12.538 \mathrm{GHz}$, respectively. At $128 \mathrm{~mA}$, only ES ML exists and one single mode beat at the cavity round trip frequency of $12.096 \mathrm{GHz}$ is 
observed. For a gain current of $132 \mathrm{~mA}$, the ES beat and the GS beat now occur at frequencies of 12.105 and $12.321 \mathrm{GHz}$, respectively. Due to dispersion, the longer wavelength of the GS results in a higher beat frequency. The beats are also transferred to a low-frequency band at $237 \mathrm{MHz}$ as shown in the inset of Fig. 2. This emission state scenario is complementary reflected in the state-resolved L-I characteristics. The experimentally obtained and modeled L-I characteristics at zero volt reverse-bias are shown in Fig. 3. The evolution of the state-resolved output power characteristics emphasizes the onset of lasing on the ES first. Then, at the onset of the GS, a

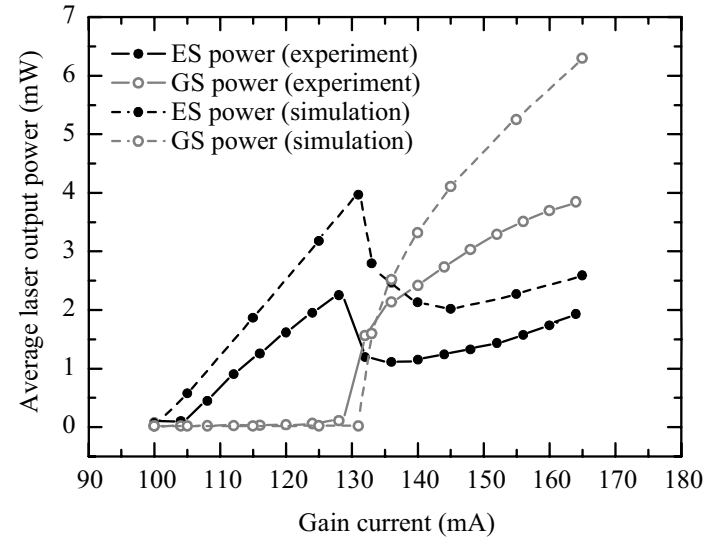

Fig. 3. Two-section QD laser reverse emission state dynamics: Spectrally resolved L-I characteristics (experimental and simulation results).

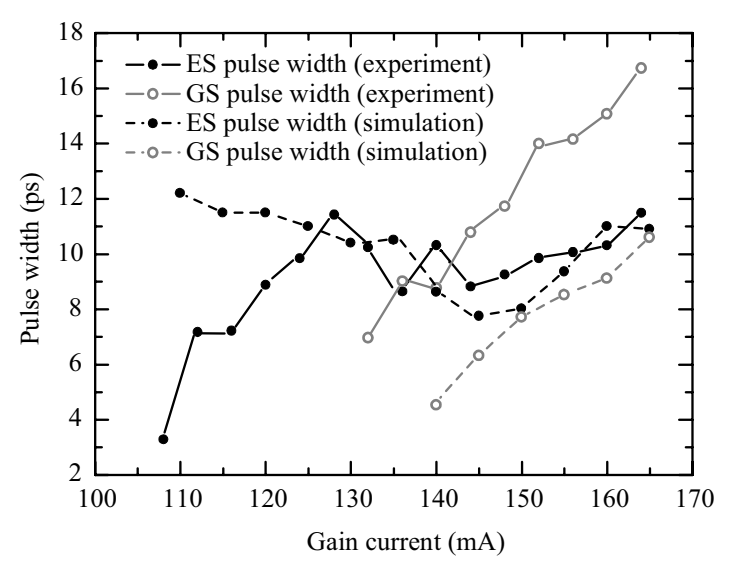

Fig. 4. Two-section QD laser reverse emission state dynamics: Spectrally resolved pulse widths (experimental and simulation results).

decrease in ES power occurs. Above a gain current of $130 \mathrm{~mA}$ both emission states contribute to the total output power. Mode-locked operation is verified by state-resolved pulse width measurements. In Fig. 4, both experimentally obtained and simulated pulse width for both ES and GS transitions are depicted. At the onset of two-state ML, shorter pulse widths are found for the GS as compared to the ES at the same gain current. Additionally, an appreciably shortening of the ES pulse widths is observed once GS ML starts. Furthermore, a huge coexistence regime of mode-locked picosecond pulses on both GS and ES is experimentally verified. Both experimentally obtained L-I and pulse widths evolution are reproduced by the modeling with an overall good agreement. These simulations allowed in particular to identify a photon-pumping or photon-recycling process in the SA section that stimulates GS emission while ES emission takes place: ES photons generated in the gain section can be absorbed in the SA section. The ES photo-generated carriers can then either be excited to higher energy states or relax into the GS while reducing GS absorption [8]. This identified physical mechanism, responsible for the novel reverse state transition, has a particularly fascinating consequence: the realization of a complete emission state control of the specifically designed multi-layer two-section QD laser by electrically shortening the SA section via an external variable resistor, forming a resistor SEED configuration [16]. By variation of the external resistor value both ES and GS carrier populations and their dynamics in the SA
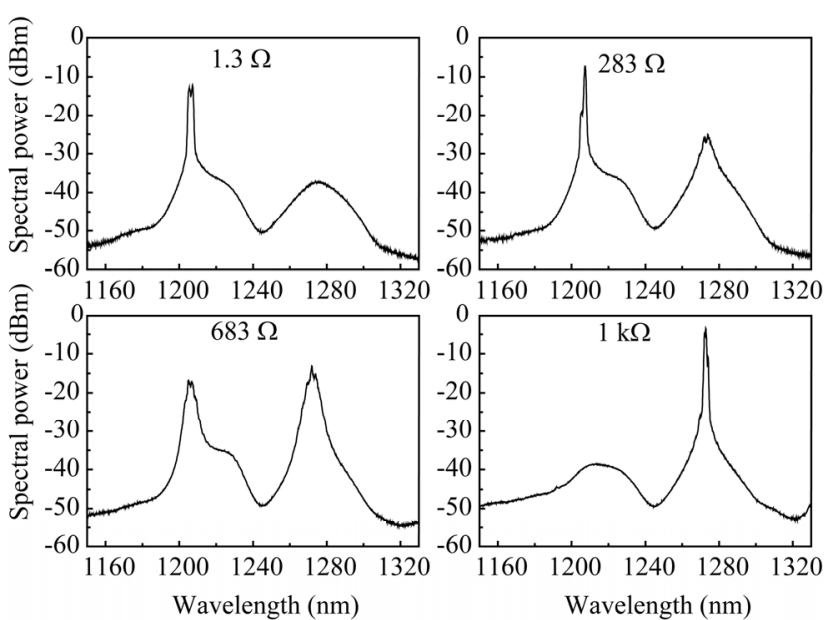

Fig. 5. Two-section QD laser absorber SEED: Optical spectra evolution, in contrary to Fig. 1, now with increasing external resistance value and at a gain current of $110 \mathrm{~mA}$ (experimental results).

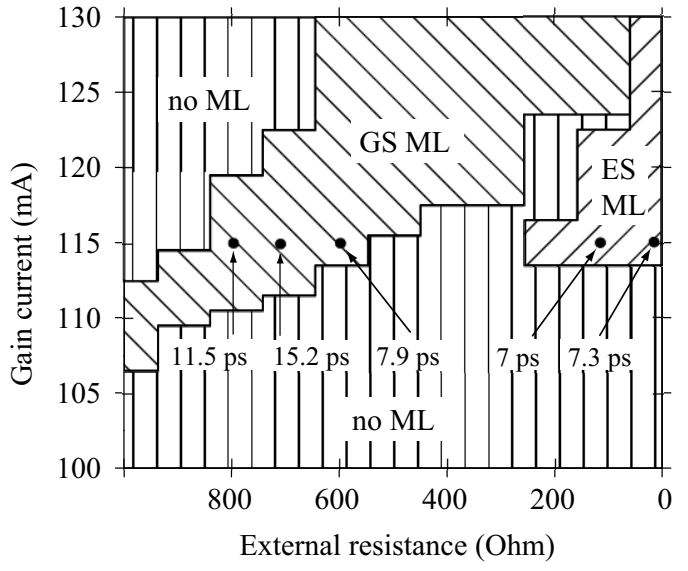

Fig. 6. Two-section QD laser absorber SEED: ML map in the plane of gain current versus external resistor value (experimental results).

section can be appreciably controlled. Experimentally obtained optical spectra at a constant gain current of $110 \mathrm{~mA}$ and at external resistance values of $1.3 \Omega, 283 \Omega, 683 \Omega$ and $1 \mathrm{k} \Omega$, respectively, are shown in Fig. 5 . At low external resistance values of $1.3 \Omega$, ES ML is observed. By increasing the external resistance value the 
photon-pumping process starts to reduce GS absorption in the SA section. At $683 \Omega$ both ES and GS exhibit lasing. By further increasing the external resistor value up to $1 \mathrm{k} \Omega$ only GS lasing and ML is obtained. This particular state-selectivity is reflected in the ML map, depicted in Fig. 6. The ML state is plotted in the plane of gain current and external resistor value. Obtained pulse widths for ES and GS, respectively, are exemplarily shown in the map for a gain current of $115 \mathrm{~mA}$.

By exploiting this resistor SEED configuration in a two-section QD laser, a novel approach to switch wavelengths of the mode-locked pulses from the ES to the GS and vice versa, over a range of $63 \mathrm{~nm}$, is demonstrated.

\section{CONCLUSIONS}

In contrary to the classical emission state transition hierarchy in QD lasers, where, with increasing gain current, GS lasing starts and, by increasing the gain current, a transition to ES lasing takes place, in this paper reverse emission state transition has been discussed for a particularly technologically designed QD structure. This reverse GS/ES state transition dynamics is observed at a saturable absorber bias of zero Volt (short circuit). A huge coexistence regime, i.e. temporal simultaneity of mode-locked picosecond pulses on two emission states, separated in wavelength by $63 \mathrm{~nm}$, is realized. Pulse width are appreciably shortened at the onset of two-state mode-locking. Time-domain travelling-wave equation modeling substantially confirm this novel operating regime and the picosecond pulse properties. The exploitation of a resistor Self-Electro-Optical Devices (SEED) configuration allow for a tailored complete emission state control and resulting wavelength-switching between ES and GS, spectrally separated $63 \mathrm{~nm}$, in a passively mode-locked InAs/InGaAs two-section QD semiconductor laser.

\section{ACKNOWLEDGEMENTS}

This work was supported by the EU Sixth Framework Program project "NANO UB-SOURCES" (Grant No. 017128) and by the EU Seventh Framework Program "FAST-DOT" (Grant No. 224338). Extremely fruitful discussions with M. A. Cataluna from the University of Dundee are most gratefully acknowledged.

\section{REFERENCES}

[1] Viktorov, E., Cataluna, M., O'Faolain, L., Krauss, T., Sibbett, W., Rafailov, E., and Mandel, P., "Dynamics of a two-state quantum dot laser with saturable absorber," Appl. Phys. Lett. 80, 121113 (2007).

[2] Gioannini, M. and Montrosset, I., "Numerical analysis of the frequency chirp in quantum-dot semiconductor lasers," IEEE J. Quant. Electron. 43(9-10), 941-949 (2007).

[3] Markus, A., Chen, J., Paranthoen, C., Fiore, A., Platz, C., and Gauthier-Lafaye, O., "Simultaneous twostate lasing in quantumdot lasers," Appl. Phys. Lett. 82, 1818-1820 (2003).

[4] Cataluna, M. A., Rafailov, E. U., McRobbie, A. D., Sibbett, W., Livshits, D. A., and Kovsh, A. R., "Ground and excited-state modelocking in a two-section quantum-dot laser," in The 18th Annual Meeting of the IEEE Lasers and Electro-Optics Society (LEOS 2005)], 870-871 (22-28 Oct 2005).

[5] Cataluna, M. A., McRobbie, A. D., Sibbett, W., Livshits, D. A., Kovsh, A. R., and Rafailov, E. U., "New mode locking regime in a quantum-dot laser: enhancement by simultaneous cw excited-state emission," in Conference on Lasers and Electro-Optics (CLEO), (21 May 2006).

[6] Cataluna, M. A., Nikitchev, D. I., Krestnikov, I., Livshits, D. A., Kovsh, A. R., and Rafailov, E. U., "Dual-wavelength modelocked GaAs-based quantum-dot laser," in European Conference on Lasers and Electro-Optics 2009 and the European Quantum Electronics Conference (CLEO Europe - EQEC 2009), 1-1 (14-19 June 2009).

[7] Breuer, S., Rossetti, M., Elsässer, W., Drzewietzki, L., Bardella, P., Montrosset, I., Krakowski,M., and Hopkinson, M., "Reverse ground-state excited-state transition dynamics in mode-locked two-section quantum dot semiconductor lasers," Opt. Lett. , submitted (2010).

[8] Breuer, S., Rossetti, M., Elsaesser,W., Drzewietzki, L., Bardella, P., Montrosset, I., Krakowski, M., Hopkinson, M., "Reverse ground-state excited-state transition dynamics in two-section quantum dot semiconductor lasers: mode-locking and stateswitching", SPIE Photonics Europe 2010, Brussels, Proc. SPIE, Vol. 7720, 772011 (2010).

[9] Miller, D. A. B., Chemla, D., Damen, T., Wood, T., Burrus, C., Gossard, A., and Wiegmann, W., "The quantum well selfelectrooptic effect device: Optoelectronic bistability and oscillation, and self-linearized modulation," IEEE J. Quant. Electron. 21(9), 1462-1476 (1985).

[10] Miller, D. A. B., "Quantum-well self-electro-optic effect devices," Opt. Quant. Electr. 22, S61-S98 (1990).

[11] Rossetti, M., Bardella, P., Montrosset, I., Breuer, S., and Elsässer, W., "Simulation and design of quantum dot lasers operating in dual-wavelength passive mode-locking regime," in 15th European Conference on Integrated Optics (ECIO2010) (2010).

[12] Ray, S. K., Groom, K. M., Beattie, M. D., Liu, H. Y., Hopkinson, M., and Hogg, R. A., "Broad-band superluminescent lightemitting diodes incorporating quantum dots in compositionally modulated quantum wells," IEEE Phot. Tech. Lett. 18(1), 5860 (2006)

[13] Rossetti, M., Bardella, P., and Montrosset, I., “A time-domain travelling-wave model including multipopulation rate-equations for passively mode-locked quantum-dot lasers," in 1st EOS Topical Meeting on Lasers, Capri, Italy, (2009).

[14] Rossetti, M., Bardella, P., Gioannini, M., and Montrosset, I., "Time domain travelling wave model for simulation of passive mode locking in quantum dot lasers," in European Conference on Lasers and Electro-Optics 2009 and the European Quantum Electronics Conference (CLEO Europe - EQEC 2009)], CF.P.2 (2009).

[15] Morton, P. A., Helkey, R. J., Bowers, J. E., Helkey, R. J., and Bowers, J. E., "Dynamic detuning in actively mode-locked semiconductor lasers," IEEE J. Quant. Electron. 25(12), 2621-2633 (1989).

[16] Breuer, S., Elsässer, W., and Hopkinson, M., "State-switched mode locking of a two-segment quantum dot laser via a selfelectro-optical quantum dot absorber," Electron. Lett. 46(2), 161-162 (2010). 